\title{
EXPOSIÇÃO DOS TRABALHOS DE CONCLUSÃO DE CURSO DO CENTRO UNIVERSITÁRIO DA FEI - INOVAFEI 2016
}

\author{
I. C. FRANCO ${ }^{1, *}$, A. M. O. SIQUEIRA,2 \\ ${ }^{1}$ Centro Universitário FEI, Departamento de Engenharia Química \\ 2 Universidade Federal de Viçosa, Departamento de Química \\ *E-mail: icfranco@fei.edu.br
}

RESUMO: A Exposição dos Trabalhos de Conclusão de Curso do Centro Universitário da FEI - INOVAFEI é um evento organizado, semestralmente, pelo Centro Universitário da FEI onde trabalhos de Engenharia de Automação e Controle, Engenharia Civil, Engenharia, Elétrica, Engenharia de Produção, Engenharia Química e Engenharia Têxtil são apresentados à comunidade acadêmica e externa. Nesta edição, a JCEC apresenta uma seleção de 17 artigos apresentados no INOVAFEI-2016 por alunos do Curso de Engenharia Química e um artigo descrevendo a história do evento.

PALAVRAS-CHAVE: Trabalho de conclusão de curso; Engenharia Química; Ensino.

\section{APRESENTAÇÃO}

O periódico The Journal of Engineering and Exact Sciences - JCEC tem como objetivo a publicação de trabalhos que ofereçam contribuição científica na área de Engenharia e Ciências Exatas e áreas afins.

Desde o ano de 2016, o periódico JCEC enfrenta um grande desafio, que é o de elevar a sua periodicidade, bem como aumentar o número de acessos à revista, com a publicação de ao menos 2 (dois) números a cada semestre e a promoção da qualificação técnico-científica de seus artigos publicados.

Nesta perspectiva, o The Journal of Engineering and Exact Sciences publica, com grande satisfação, nesta edição, organizada pelo Prof. Dr. Ivan Carlos Franco, 17 artigos previamente apresentados durante o evento INOVAFEI 2016 pelos alunos do Curso de Engenharia Química do Centro Universitário da FEI e um artigo apresentando o evento e relacionando-o com os Trabalhos de Conclusão de Curso. Os trabalhos são listados a seguir e na Tabela 1 são apresentados os respectivos avaliadores. 
1. INOVAFEI $E$ OS TRABALHOS DE CONCLUSÃO DE CURSO DE ENGENHARIA QUÍMICA DO CENTRO UNIVERSITÁRIO DA FEI

Ricardo Belchior Tôrres, Ivan Carlos Franco e Luis Fernando Novazzi

2. PRODUÇÃO DE RESINA TROCADORA DE ÍONS A PARTIR DE POLIETILENO RETICULADO

Camila Figueiredo de Castro, Luz Consuelo Gonzalez Alonso Panzarini, Maggiory Simões Ferrara, Marcela Pelegrino Marques, Maria Fernanda Ferraz de Almeida, Tarsilia Matos Machado e Yuli Silvino Capelle

3. ESTUDO DA EXTRAÇÃO DE CORANTE NATURAL QUE CONFERE PROTEÇÃO ULTRAVIOLETA EM FIBRAS NATURAIS

Adriana Célia Lucarini, Alex Sthefan Franco Zelada Tabu, Felipe de Freitas Roque da Silva, Gabriela de Andrade Moraes, Gabriela Iervolino Piazon e Mariana Pimenta Zurawski

4. ESTUDO DA REMOÇÃO DE INIBIDORES DE FERMENTAÇÃO APÓS TRATAMENTO ÁCIDO E ENZIMÁTICO DE PALHA DE CANA

Adriana Célia Lucarini, Newton Libânio Ferreira, Charles Douglas de Souza, Felippe Pinheiro Lima Francisco, Isabela Reinaud Fonseca, Larissa Guedes Rossi e Vanuza Magalhães Ferreira

5. COMPARAÇÃO ENERGÉTICA ENTRE OS PROCESSOS DE OBTENÇÃO DE AROMÁTICOS DE MISTURAS DE HIDROCARBONETOS RICAS EM AROMÁTICOS

Aline Zacarelli, Caroline Alves Sampaio Faria, Jéssica Amorim Matos, Kemer Neves Zuri, Leonardo Seiji Otsubo Hayasida e Newton Libânio Ferreira

6. PROJETO DE INSTALAÇÃO DE PRODUÇÃO INDUSTRIAL DE CARBONATO DE GLICERINA

Beatriz Peragine Celiberto, Caio Canale Fico, Guilherme Russo, Luccas Lossano Name, Victor Manuel Betancourt Barros e Newton Libânio Ferreira

7. INFLUÊNCIA DE LÍQUIDOS IÔNICOS NO TRATAMENTO DA PALHA DE MILHO PARA A PRODUÇÃO DE BIOETANOL

Andreia de Araújo Morandim-Gianetti, Patrícia dos Reis Campos, André Viana, Rafaela Paz Trujillo Martinez, Márcio Dominato, Rosianne Cristina Silva e Murilo Albuixech Santoro

8. ESTUDO DO USO DA LIGNINA-PEROXIDASE PARA REMOÇÃO DE 2CLOROFENOL EM EFLUENTE LÍQUIDO 
Juliana Mendes de Oliveira, Andreia de Araújo Morandim-Gianetti e Adriana Célia Lucarini

\section{PRODUÇÃO DE BIODIESEL A PARTIR DO ÓLEO DE PALMISTE}

Adriana Célia Lucarini, Newton Libânio Ferreira, Aline Miyuki Natsumeda, Camilla Brabo de Aguiar, Caroline Cassian Souza e Fernanda Larelio

10. ANÁlISE DE RISCOS E CONSEQUENCIAS EM UMA USINA SUCROALCOOLEIRA

Martisthela Passoni de Araújo Marin, Gabriela Messias de Melo, Guilherme Barbosa Henke de Paulo, Luiz Fernando Machado Labate, Thiago Baco Miranda e Vanessa Schincariol

11. APLICAÇÃO DO PROCESSO FENTON PARA DESCONTAMINAÇÃO DE HIDROCARBONETOS AROMÁTICOS EM ÁGUA

Felipe Ayoub Zanini, Fernando Augusto Romero dos Santos, Leandro Akira Matsumura, Luis Fernando Novazzi, Mariana Toze Francisco e Tais Macedo Paiva

12. ESTUDO DA UTILIZAÇÃO DE SOLVENTE ALTERNATIVO NO PROCESSO DE EXTRAÇÃO DO ÓLEO DE SOJA

Denise Borsos Baião, Bianca Screpante Garé, Ana Carolina Mauro Ribeiro, Nathan Guola Trovão e Rodrigo Condotta

13. ESTUDO DO PROCESSO DE SECAGEM DA LINHAÇA EM LEITO FLUIDIZADO E EM LEITO DE JORRO

Diogo Roberto Arrotéia, Beatriz Gimenez Barbalho, Guilherme Caetano Cara, Vinícius Risóleo, Letícia Canha dos Santos, Fernado Abbes Gurgel Testa e Rodrigo Condotta

14. ELIMINAÇÃO DE COMPOSTOS ORGÂNICOS VOLÁTEIS POR MEIO DE ABSORÇÃO EM ÓLEO EM COLUNAS DE ENCHIMENTO

Luciano Gonçalves Ribeiro, Henrique da Silva Monteiro, Nickolas Scarpelli Denardi, Willy Hung Hsu e Yuri Cazzaro Rocha

15. ESTUDO DA HIDRÓLISE ENZIMÁTICA DA PALHA DA CANA DE AÇÚCAR PARA PRODUÇÃO DE ETANOL DE SEGUNDA GERAÇÃO

Adriana Célia Lucarini, Ana Carolina Tezotto Delquiaro, Ludmila Carolina Prado Tavares Vidoca, Rafaela Braz, Roberta Morelli Martins e Taís Pereira Alves

16. ANÁliSE ESTATÍSTICA APLICADA AOS PARÂMETROS DE SÍNTESE DE FAUJASITA Na-X 
Mayara dos Santos Castro, Wellina Massassi Fantim, Rodrigo Condotta, Eliezer Ladeia Gomes e João Guilherme da Rocha Poço

\section{TRATAMENTO DE EFLUENTES DE CURTUME A PARTIR DA ADSORÇÃO EM QUITOSANA SUBMETIDA A TRATAMENTO COM LÍQUIDO IÔNICO}

Andreia Araújo Morandim-Giannetti, Kevy Pontes Eliodorio, Vitor Seorra Andolfatto, Marco Rulevas Martins, Breno Pivaro de Sá e Erick Umeki

18. ANÁLISE E OTIMIZAÇÃO DAS CONDIÇÕES OPERACIONAIS DE UM REATOR DE LEITO FIXO NA SÍNTESE DE FISCHER-TROPSCH

João Vitor Trabbold Delbianco, Lucas Francisco M. C de Oliveira, Lucas Vilas Boas e Luis Fernando Novazzi

Tabela 1 - Relação de avaliadores dos trabalhos apresentados no INOVAFEI 2016

\begin{aligned} & \hline Avaliadores \\ & \hline 1 Adriana Célia Lucarini (Centro Universitário da FEI) \\ & 2 Daniel Benestante Hauk (Centro Universitário da FEI) \\ & 3 Fernanda Guerreiro Rossi Higa (Centro Universitário da FEI) \\ & 4 Ivan Carlos Franco (Centro Universitário da FEI) \\ & 5 João Guilherme da Rocha Poço (Centro Universitário da FEI) \\ & 6 Luis Fernando Novazzi (Centro Universitário da FEI) \\ & 7 Luz Consuelo Gonzalez Alonso Panzarini (Centro Universitário da FEI) \\ & 8 Maristhela Passoni de Araújo Marin (Centro Universitário da FEI) \\ & 9 Newton Libânio Ferreira (Centro Universitário da FEI) \\ & 10 Ricardo Belchior Tôrres (Centro Universitário da FEI) \\ & 11 Rodrigo Cella (Centro Universitário da FEI) \\ & 12 Rodrigo Condotta (Centro Universitário da FEI) \\ & \hline\end{aligned}

Desta forma, a JCEC sente-se honrada por ser um importante canal de comunicação científica, e neste número v. 3, n 2 (2017), o periódico disponibiliza a toda sociedade, trabalhos originais, com temas relevantes de interesse científico e tecnológico para o desenvolvimento socioeconômico na área de Engenharia Química. O corpo editorial espera que os leitores aproveitem ao máximo o conhecimento publicado nesta edição.

The Journal of Engineering and Exact Sciences - JCEC Journal of Chemical Engineering and Chemistry 